\title{
Industrial Application of Point Cloud / STL DATA FOR REVERSE ENGINEERING
}

\author{
Kumar, A.; Jain, P. K. \& PATHAK, P. M.
}

Abstract: This chapter presents the state of art for the application of generated point cloud/Stereolithugraphy (STL) data in industry. Generated point cloud and (STL) is emerging as a representation format for geometric modelling. STL is the standard data format used in rapid prototype and reverse engineering methodologies using triangular facet with a normal vector pointing away from the surface in the solid. The point cloud data is a dense set of discrete coordinate points and samplings from the surface of a three dimensional scanned object. Point cloud and STL data are scanning based advance file format associated with the contact or non contact scanning device. Generation of point cloud and STL data is used as productive techniques increase the accuracy quality and to control noise of scanned model. The present chapter present a detailed description of process methodology, methods of scanning, applications, advantages and limitations on point cloud and STL. Further, the adaptive wear out area of gear tooth has been determined using reverse engineering approach. Finally, the technique was demonstrated to scan the existing physical object in order to improve the product quality by the applications of CAD/CAM/CAE techniques.

Key words: reverse engineering, scanning techniques, point cloud data, STL format
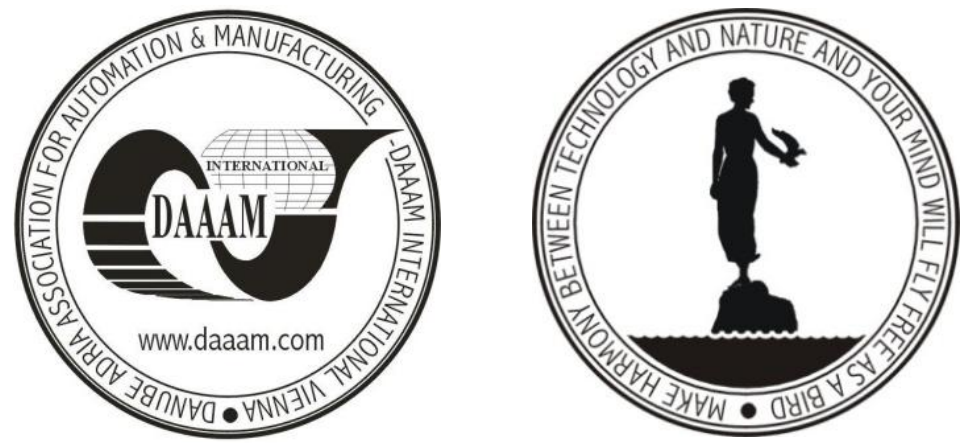

Authors' data: Kumar, [Atul], Jain, P[ramod] K[umar]; Pathak, P[ushparaj] M[ani], Mechanical \& Industrial Engineering Department, Indian Institute of Technology Roorkee, India, atulkumar.iitr@gmail.com, pjainfme@iitr.ernet.in,pushppathak@gmail.com

This Publication has to be referred as: Kumar, [Atul]; Jain, P[ramod] K[umar] \& Pathak, P[ushparaj] M[ani] (2012). Industrial Application of Point Cloud / STL Data for Reverse Engineering, Chapter 38 in DAAAM International Scientific Book 2012, pp. 445-462, B. Katalinic (Ed.), Published by DAAAM International, ISBN 978-3-90150986-5, ISSN 1726-9687, Vienna, Austria

DOI:10.2507/daaam.scibook.2012.38 
Kumar, A.; Jain, P. K. \& Pathak, P. M.: Industrial Application of Point Cloud / ST...

\section{Introduction}

Due to an accelerated pace of change in technology, reverse engineering (RE) is a growing field that continues to go forward to suit the rapid changes of the 21st century. Engineering fields are constantly improving upon current designs and methods to make life simple and easier. Engineering can be defined as "the application of scientific and mathematical principles to practical ends such as the design, manufacture, operation of efficient and economical machines, structures, processes, maintaining product and systems". There are two ways to accomplish this: forward engineering and reverse engineering. Forward engineering is the traditional process of moving from high-level abstractions and logical designs to the physical implementation of a system. In some situations, there may be a physical part product without any technical details, such as drawings data, bills-of-material, etc. The process of duplicating an existing part or subassembly of product (without drawings, and documentation) on a computer is known as reverse engineering. Reverse engineering provides a problem's solution by the physical model, which is the source of information for the CAD model. This is also referred as the conversion of physical-to-digital word shown in figure 1. Another reason for reverse engineering is to compress product development cycle times. In competitive global market, manufacturers are constantly seeking new ways to shorten lead times to market a new product. There has been a mandatory need of shorten the product devlopment time and analyse the objects of the manufacturing industry, medical industry, military branches and research facilities which is fullfilled by reverse engineeirng approch. Manufacturing industry use reverse engineering to insure fast rapid prototyping ability and accuracy associated with the production of new parts. Rapid product development (RPD) refers to recently developed technology and techniques that assist manufacturers and designers in meeting the demands of shortened product development time.

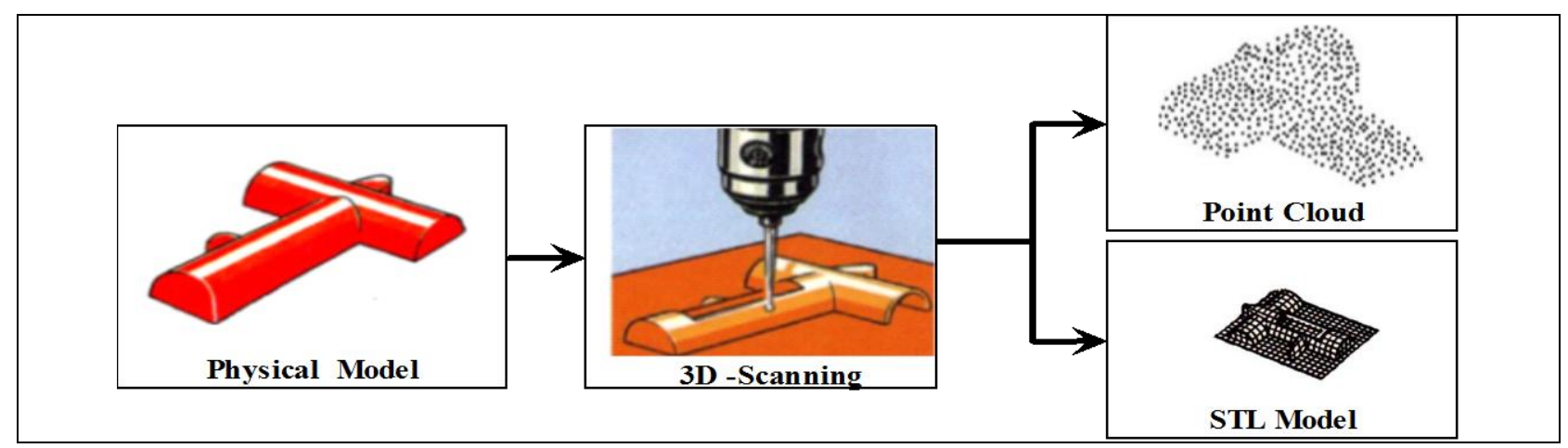

Fig. 1. Physical-to-digital process (Sokovic \& Kopac, 2006)

The objective of reverse engineering is to reconstruct updated mathematical geometric models from existing physical object. The appropriate utilize scanning techniques (contact or non-contact), CAD/CAE/CAM technology and rapid prototype approach with significantly to obtain an optimal design with reduces product development time (Liang \& Grier, 2000). 


\section{Reverse Engineering Methodology}

In recent years, extensive attention has been focused on different methodologies of reverse engineeirng The state of the art in reverse engineering is to reconstruct true geomatric shape from physical objects in an efficent and acquate way (Liang \& Grier, 2000). The characterizes typical procedure of reverse engineering shown in figure 2. It consists of five steps: (1) data acquisition, (2) preprocessing (noise filtering and merging), (3) triangulation, (4) feature extraction, and (5) segmentation and surface fitting (Bidanda \& hosni, 1994; Chang \& Park, 1994).

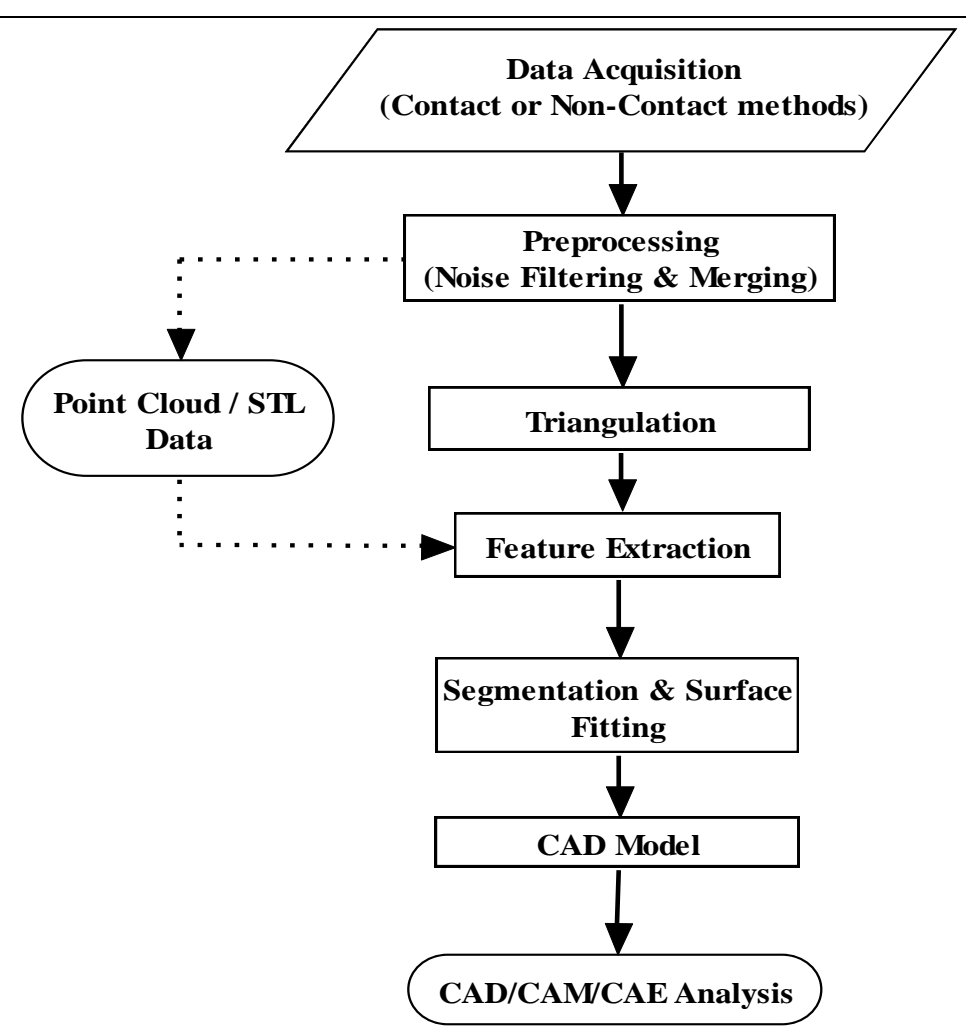

Fig. 2. Typical hierarchy of reverse engineeirng

Data acquisition and processing systems includes hardware and software components. A hardware system acquires point clouds or volumetric data by using available experimental setup. A software system processes raw point clouds or volumetric data and transfers them into a virtual representation of object surfaces. The point cloud data is acquired in the form of $\mathrm{x}, \mathrm{y}$ and $\mathrm{z}$ co-ordinates of the multiple point of the object surface (Bi \& Wang, 2010). The scanning techniques use to scan the object are contact and noncontact technique. These techniques are discussed in details in next section. 3D digitization system such as non-contact 3D scanner generated the large amount of data. In general, scanning data can be saved in different file formats, out of which the point cloud and STL formats are very usefull for research assessment (Woo et al., 2002), (Hur et al., 2011; Yang et al., 2011). Point cloud and STL data are most widely known notorious issues in reverse engineering. Noise is everywhere in measured data. Therefore, before the surface reconstruction process it is necessary to prepare the noise free scanned data. This 
preparation is frequently described as preprocessing. In digitization system, surface reconstruction methods and pre-processing phase can entail different processes. For the pre-processing the steps involved are: noise filtering, data smoothing and data reduction. The segmentation process splits a triangular mesh into sub-meshes to which an appropriate single surface can be fitted, and it seriously affects the quality of the resulting CAD model. To improve the quality of segmentation, it is essential to make the use of features (sharp edges and symmetry planes) extracted in the fourth step. Among various features, few features are very important for the segmentation process because of them, some feature properties are directly appled in industrial products. A concept with redesign and prototyping of free-form surfaces in the manufacturing industry often involves. The application of physical models for initial conceptual and aesthetic design, product prototyping, and performance testing and model modification are needed for obtained an optimal product design. ComputerAided Design (CAD) is the use of computer technology to assist in the design particularly in technical drawing and engineering drawing of an object. CAD is mainly using for detailed engineering of 3D models and 2D drawings of physical components, but it is also used through out the reverse engineering process from $3 \mathrm{D}$ models and 2D drawings of physical components which do not have available technical drawing and engineering drawing (Tai \& Hung, 2000 ; Hussain et al., 2008). In contrast to the tradinatioal production sequence after dizitigation the existing objects, a solid model or surface model can be deduced in order to make use $\mathrm{CAD} / \mathrm{CAM} / \mathrm{CAE}$ technologies to full fill the modren industry demand.

\subsection{Reverse Engineering for Rapid Prototyping}

The 3D-digitising and reconstruction of 3D-shapes by reverse engineering has numerous applications due to which it is an interesting research and development field relating the reverse engineering to rapid prototyping as shown in figure 3.

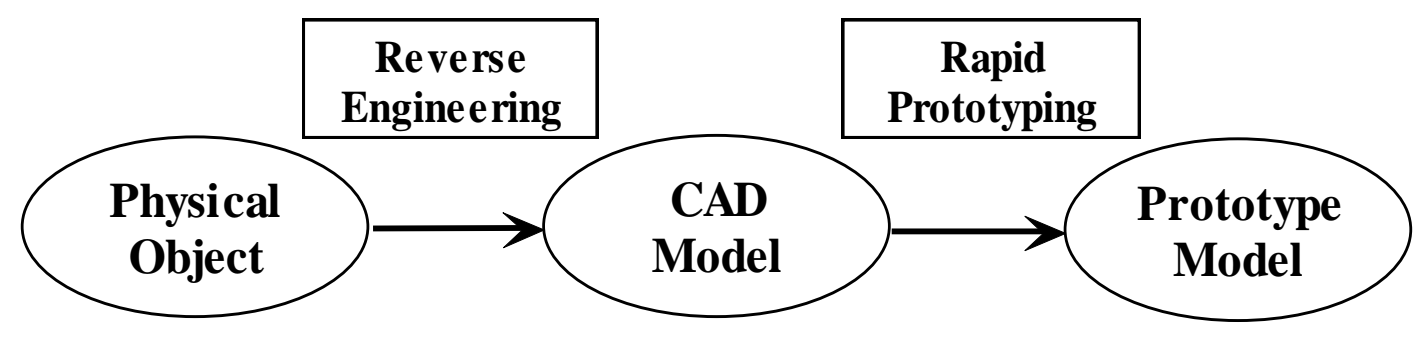

Fig. 3. Relationship between reverse engineering and rapid prototyping

Rapid prototyping provides the mean to reduce the lead time required to produce a physical prototype of an object. Rapid prototype technique build the model, one layer at a time, from bottom to top. While most traditional manufacturing techniques use subtractive or formative processes. The additive nature provides cohesiveness with the data types created by reverse engineering systems. Once the data has been successfully acquired by the reverse engineering system, limitless design and alternatives are achieved through modification or scaling of the newlyacquired $\mathrm{CAD}$ data which can be further used to produce physical prototypes to 
achieve manufacturing objectives. The practical integration of contact and noncontact reverse engineering and rapid prototyping techniques is becoming more and more prevalent each year. The related fields are engineering, automotive, medical, aerospace, entertainment, electronics, and consumer goods and take advantage of easily integrated technologies to develop creative solutions of design problems.

\subsection{Reverse Engineering for Rapid Product Development}

The reverse engineering is now an accepted technique of contemporary product design and manufacturing process. The reverse engineering process can be loosely defined as a process that creation of a results in the mathematical model from a physical model. Nowadays the management of engineering product design could be realised based on the two methodologies called "conventional approach" and "nonconventional approach". In conventional approach the products design is start from the ground stage with the geometric modelling and utilize CAD/CAE/CAM techniques. The geometric model could be represented as surfaces or as a solid model. The conceptual model generated CAD information could be exported subsequently in standard format like IGES, point cloud, STL, binary and ASCII data and imported in the same data format to CAE/CAM techniques. The product development by conventional approach is not applicable when the goal is to reengineer or to simulate and to optimise parts/moulds/tools already exist without design information in CAD data format. Consequently, this will be necessary to apply techniques that allow capturing the geometry of parts/moulds/tools (or prototypes), and to generate a conceptual 3D model that will be used in CAE and CAM systems. This process is regularly called reverse engineering under non-conventional approach (Sokovic \& Kopac, 2006).

\subsection{Reverse Engineering Applications}

It is evident from the principle method and literature review of reverse engineering in point cloud/ STL data. It has many benefits over forward engineering for the CAD model. The manufacturing industry have wide area to uses, scanned data by means of reverse engineering to fast process and reduces the overall product development time. In figure 4 shows the complete cycle for individual reverse engineering applications, their key reason and brief description of applications is given below:

- New Design: The design process of a new product does not always start from a CAD model. A prototype is often built first. Once the design is approved, measurements are made (either manually or with the use of a contact probe). The extracted data are then manually entered into a CAE system for further analysis. This process has two disadvantages: it is time-consuming and a potential source of measurement errors.

- Modify Existing Design: In some instances the design of an existing product must be modified. The modification process and design improvements are best performed on a CAD model. However, CAD models for many existing products are not available. Part image reconstruction systems can play an important role in reducing design time. 
- Industrial Inspection: When a part is compared with its existing CAD model, a reverse engineering system can acquire the actual map of the part surface, and deviations, if any, can be identified.

- Design of Large Items: Precise measurements of large parts are often not possible with traditional metrological equipment. Reverse engineering-based part image reconstruction systems can help by mapping the part surface in the form of CAD model. This CAD model can now be scaled and modified as needed.

- Worn or Broken Parts: When a part breaks or worn and the engineering drawing is no longer available, part image reconstruction systems can be used to create the CAD model. The CAD model can now be used to manufacture the clone of the worn or broken part.

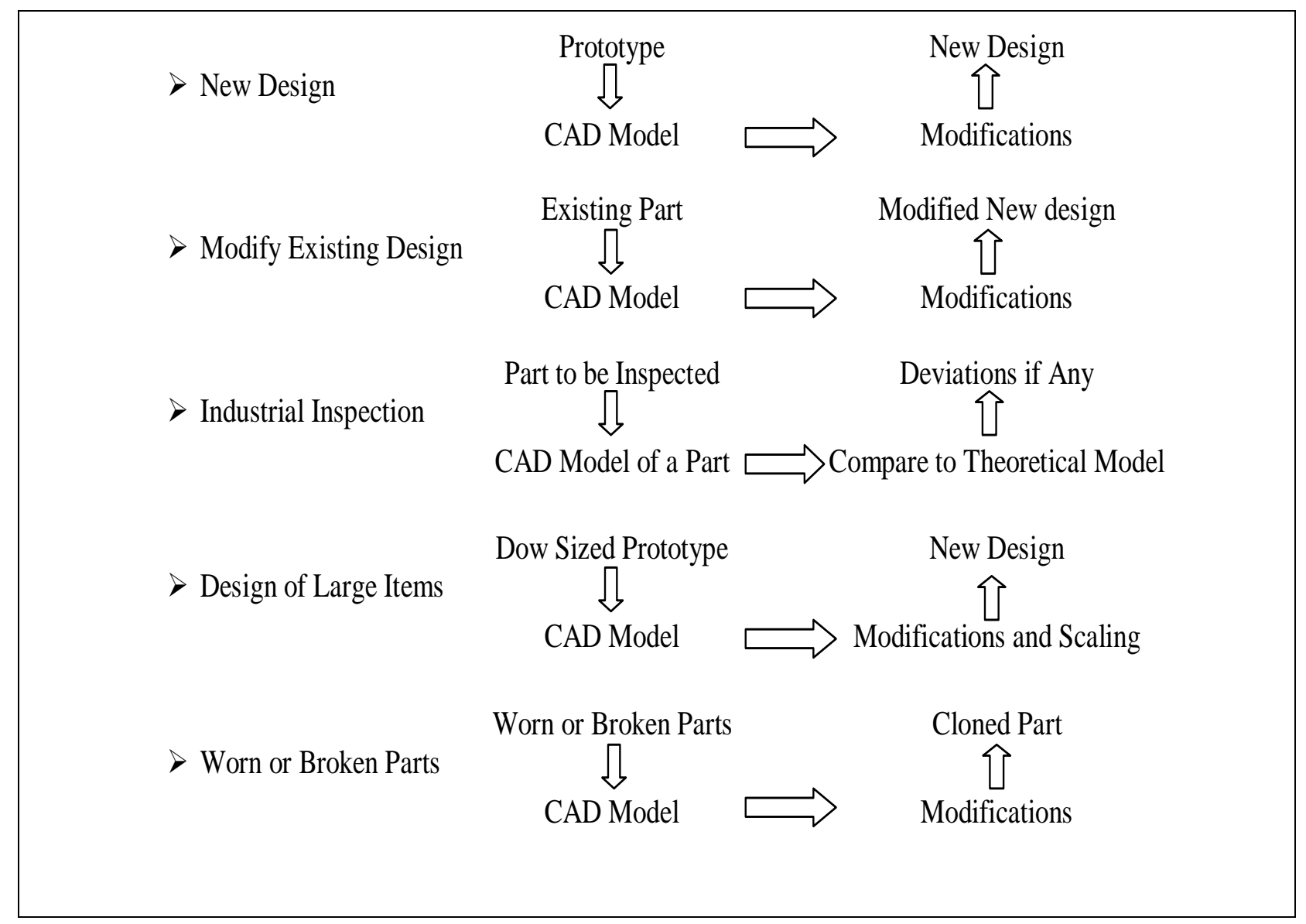

Fig. 4. The sequence of steps requierd for the application of reverse engineeirng

\subsection{Advantages and Limitations in Reverse Engineering}

The modern automotive industry reverse engineering is frequently use over other manufacturing techniques. The main and foremost advantages of reverse engineering are fast availability of CAD models and physical model is used as the starting point, shortened development process, fully developed product at the start of production and reduction in product and production costs. To complete the reverse engineering process the better methods are needed for deciding quality of sensor. Open problems remain in freeform surfaces combining with manufacturing features, particularly with regards to segmentation and surface blending. 


\section{Previous Research}

During the last decade, number of reverse engineering methodologies has been developed. In 21 century, these methodologies have been change rapidly due to gradually changing in technologies. When refine to technology, it become simple and ease that be directly related to fast and accurate. In this section a brief review of the state-of-the-art in reverse engineering in general definition, methodology, digitization technique, point cloud data, STL, noise reduction, CAD model, surfaces creation, geometry error analysis, estimate the out portion, curve patches and FEM analysis.

Raja \& Fernandes (2008) define the engineering as, the process of designing, manufacturing, assembling, and maintaining products and systems. There are two types of engineering, forward engineering and reverse engineering. Forward engineering is the traditional process of moving from high-level abstractions and logical designs to the physical implementation of a system. The process of duplicating an existing part, subassembly, or product, without drawings, documentation, or a computer model is known as reverse engineering. Tong et al. (2008) explained that reverse engineering is a digital technique developed for setting 3D CAD models from physical objects, which is an effective method for measuring complex surfaces. The output of this phase is geometric model in one of the proprietary formats such as point cloud, IGES, VDA, STL, DXF, OBJ, VRML, ISO G Code, etc. Bopaya \& Hosni (1994) defined the objective of any reverse engineering approach is to generate a 3-D mapping of the product in a CAD file format. In order to do this, the $\mathrm{x}, \mathrm{y}, \mathrm{z}$ coordinates of multiple points on the product surface. These coordinates can be used to develop the drawing of product further for redesign or CAD/CAM packages. Durupt et al. (2010) focused on reverse engineering in mechanical design and proposed the Knowledge Based Reverse Engineering methodology, which allow managing and fitting manufacturing and functional features. Zhoul et al. (2008) introduced the imaging measurement system for rubbing parts. The rubbing part model was constructed based on an Amsler friction and wear test machine. Image process technology was applied to characterize the images of adhesive wear and abrasive wear. Wear images were first enhanced by median filtering method then a reiterative algorithm was used to compute the segmenting thresholds. Gao et al. (2006) proposed a defects-free model-based repair strategy to generate correct tool paths for build up process and machining process adaptive to each worn component through the reverse engineering application. Based on 3D scanning data, a polygonal modeling approach is introduced to rapidly restore worn out parts use for welding, machining and inspection processes. Bagci (2009) discussed about obtaining the $\mathrm{CAD}$ data from damaged three different parts to reproduce or make a redesign for component, whose technical drawings is not available. The CAD models can be recovered, reconstructed and considering with parametric and geometric continuity. These evils have been solved by referring to some practical approaches and establishing continuity across curve and surface patches for free form surface modeling. Tsakatikas \& Kaisarlis (2007) presented an investigation of the criticality analysis applied for the classification of industrial spare parts, in conjunction with the implementation of reverse engineering techniques. 
Kumar, A.; Jain, P. K. \& Pathak, P. M.: Industrial Application of Point Cloud / ST...

Based on for prioritizing, the need for re-engineering components for maintenance purposes and the equipment criticality are use for an adapted failure mode effects and criticality analysis technique based on the operation and failures history record. Gadelmawla (2011) had utilized the computer vision technology to develop a noncontact and rapid measurement system for measuring and inspecting most of spur gear parameters with an appropriate accuracy. A vision system has been established and used to capture images for gears to be measured or inspected processes using Microsoft Visual. Tut et al. (2010) presented some aspects about design using $\mathrm{CAD} / \mathrm{CAM} / \mathrm{FEA}$ programs with rapid prototyping, scanning and measuring machining and its integration in industrial field. A new gasket for a ball screw used in a bending tube machine was produced by rapid prototyping techniques starting from a broken one. First the broken gasket was scanned by scanning machine obtaining the primary $3 \mathrm{D}$ model which is imported to $\mathrm{CAD} / \mathrm{CAM}$ programs and the final product is achieved on milling machine. The gasket mechanical characteristics were investigated by finite element analysis. After FEA simulation, a new material was chosen in order to increase the mechanical characteristics and also improves the tool, wear, life, scuff resistance and handling characteristic. A safety stock of the thus defined critical components is of paramount importance and must be readily available. In order to achieve new material properties, especially in the case of mechanical equipments are considering obsolete, remanufacturing through reverse engineering techniques. It must be considered one of the most important reverse engineering tasks is the assignment of the dimensional and geometrical accuracy specifications of the reverse engineered components that are directly connected with manufacturing cost and time. Today the automotive manufacturing industries with reverse engineering produce the fully developed product at the start of production so they can be reduce the cost of product and production.

\section{Classification of Digitization Techniques}

The first objective of reverse engineering methodology is to digitize the physical model. Digitization is the process of capturing the data of the physical model and converting digital form. It can be achieved by utilizing either contact probing or non-contact sensing techniques. Figure 5 classified the types of allication used for acquiring 3D data into contact and non-contact methodes.

\subsection{Contact Data Acquisition Techniques}

There are many different methods for acquiring the data, as shown in Figure 5. Out of them tactile or contact methods are represent as popular approach to shape capture. In tactile, two most commonly known forms are Coordinate Measuring Machines (CMMs) with mechanical or robotic arms with a touch probe sensing device and $3 \mathrm{D}$ laser scanner. CMMs and laser scanner both are often used when high precise measurement is required. It is considered a contact type method that is NCdriven and can be programmed for sampling of points for predefined features (Lee et al., 2001). 


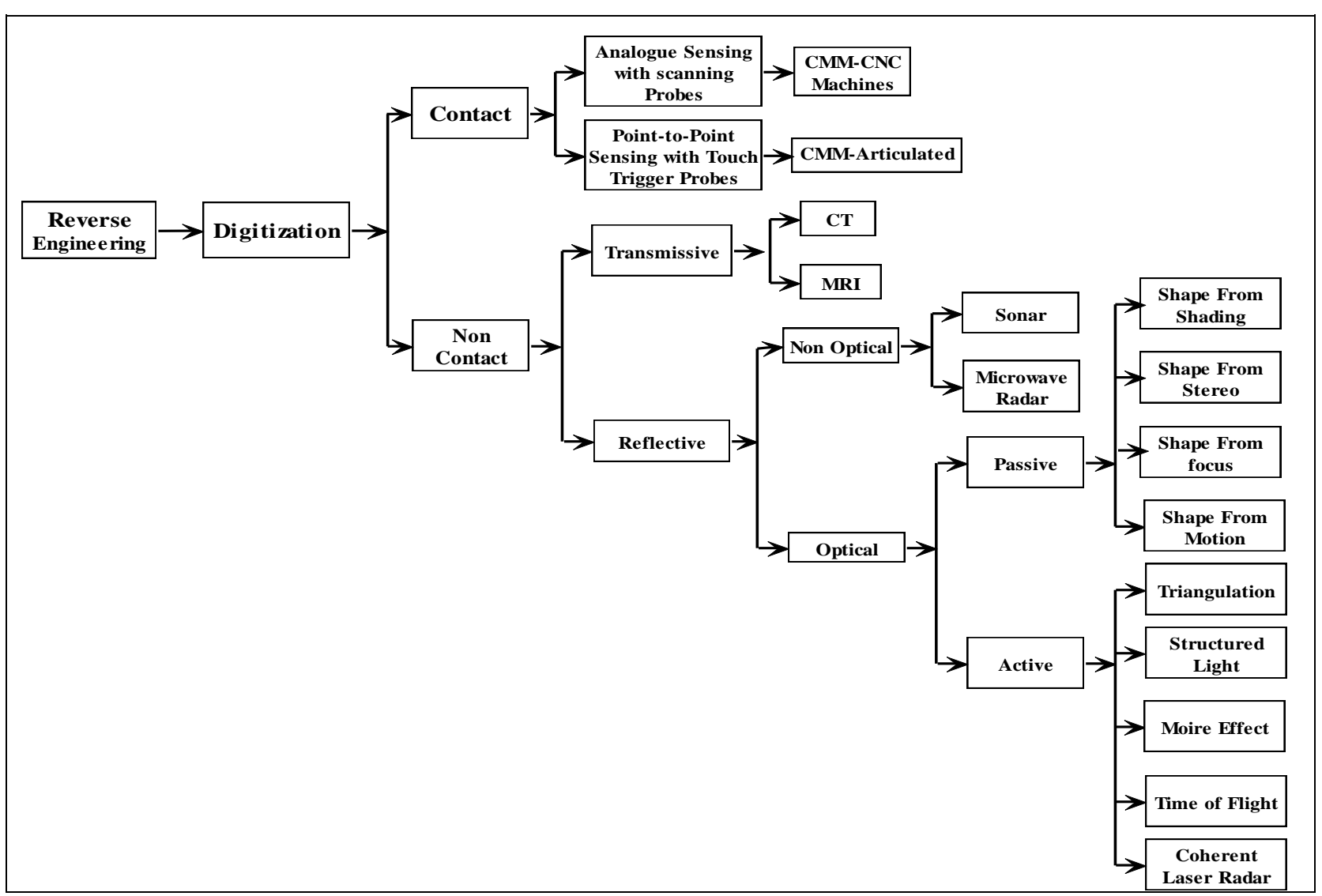

Fig. 5. Classifications of data acquisition techniques used in contact and non contact approches for Revere Engineeirng technique

These machines can be programmed as to follow define paths along a surface and collect very accurate, nearly noise-free data. The in depth discussion of measurement and profile error in tactile measurement was given by Xiong (1990). Butler (1991) provides a comparison of tactile methods and their performance. For tactile methods, geometric complexity increases the number of points required for accurate measurements. Sahoo \& Menq (1991) used the tactile systems for sensing complex sculptured surfaces. The time needed to capture the points, one by one can range from days or sometimes weeks for complicated parts. An external factor also affects the accuracy of the scanning techniques. The main factors are temperature, vibration and humidity. The main disadvantage of tactile methods is the damage an object surface and change in object surface profile (Bardell et al., 2003). However, tactile measurement, remains of a very becomes a very powerful part of measuring process in spite of above trained and disadvantages. The CMMs use touch-trigger probe of Cartesian robot as well as surface and boundary continuous probing or scanning of parts. It may also be used for the extraction of geometric feature data from points cloud or STL data.

\subsection{Non Contact Data Acquisition Techniques}

The scanning type CMMs can capture the more sampling point than the touch trigger-type and have better accuracy than vision sensors. As per discussed, each method has strengths and weaknesses that require the data acquisition system to be carefully selected for the shape capture functionality desired. As per discussion the 
Kumar, A.; Jain, P. K. \& Pathak, P. M.: Industrial Application of Point Cloud / ST...

tactile method cannot measure the parts made of soft materials and have relatively lower scanning speed compared to non-contact techniques. Non-contact methods use light, sound or magnetic fields to acquire shape from objects. In the case of contact and non-contact, an appropriate analysis must be performed to determine the positions of the points on the objects surface.

Optical methods of shape capture are probably the broadest and growing in popularity over contact methods. There are five important categories of optical methods: laser triangulation, time-of-flight, interferometers, structured lighting and stereo analysis. Active methods are distinguished from passive methods in that artificial light is used in the acquisition of data. This is because they have relatively fast acquisition rates. Correlation of image pairs and landmarks within the images are big difficulties with this method and because of that active methods are preferred. Laser Triangulation is a method, which uses location and angles between light sources and photo sensing devices to deduce position. A high-energy light source is focused and projected at a pre-specified angle at the surface of interest. A photosensitive device, usually a video camera, senses the reflection of the surface and then by using geometric triangulation from the known angle and distances. Triangulation can acquire data at very fast rates. The accuracy is determined by the resolution of the photosensitive device and the distance between the surface and the scanner. Moss et al. (1989) present a detailed discussion of a classic laser triangulation system used to capture shape data from facial surfaces. The use of laser triangulation on a coordinate measuring machine is presented by Modjarred (1998). These references give a broad survey of methods, approaches and limitations of triangulation. Measuring distance by sensing time-of-flight of the light beams emitted is the way a ranging system works. Practical methods are usually based on lasers and pulsating beams. Jarvis (1983) presents an in-depth article on time-of- flight range finders giving detailed results and analysis. Structured lighting involves projecting patterns of light upon a surface of interest and capturing an image of the resulting pattern as reflected by the surface. The image must then be analyzed to determine coordinates of data points on the surface. A popular method of structured lighting is shadow Moire, where an interference pattern is projected onto a surface producing lighted contour lines. These contour lines are captured in an image and are analyze to determine distances between the lines. The final optical shape capture method of interest is stereo image analysis. This is similar to structured lighting methods in that frames are analyzed to determine coordinate data. This method is often referred to as a passive method since no structured lighting is used. One more data acquisition methods will examine as an acoustic, where sound is reflected from a surface, magnetic, where a magnetic field touches the surface and a hybrid of both contact and non-contact. Sonar is used extensively for decades for distance measuring. In mentioned contest, the reverse engineering with contact or non-contact digitization technique are absolutely necessary because allow capturing and digitization the $3 \mathrm{D}$ object geometry to be utilized in generating line, curve, surfaces, CAD/CAM/CAE techniques, tool path generation etc. Therefore, reverse engineering is generated the numerical simulation of the process and product optimization to increases the final product quality using captured and digitized 3D object geometry. 


\subsection{Hybrid Techniques Acquisition Techniques}

Hybrid modeling systems are a combination of contact and non-contact systems or NC coding and laser scanning techniques (Clark 2000). The first type of hybrid is usually consists of the coordinate measuring machine and integrated laser based technology. The technique focuses on modeling complex and free-form shapes of mechanical objects by comparing contact and non-contact methods for digitizing the surface. During hybrid scanning the effects of ambient lighting are discussed for non-contact systems. Whether or not the system can measure the ambient lighting depends on the projected color of light on the object. If a system projects laser light then the unwanted frequencies can be filtered out. If the system projects white light, then no particular frequencies can be blocked out. This is because it might be carrying the information required to measure the object. Therefore, white light area based systems will be limited in their ability to measure ambient lighting verses laser based systems.

\subsection{Practical Problems of Data Acquisition Techniques}

In order to meet the requirements, a large number of difrent (Contact or NonContact) 3D digitization systems has been developed, among the most prominent are Coordinate masuering machine ( CMM), 3D laser scanner, pntograph, CCD cameras, computer tomograpy (CT), etc. When acquaring the data, these techniques have some practical problems such as (Varady et al. 1997):

- Calibration: Calibration is an essential part of setting up and operating a position-measuring device. Systematic sensing errors can occur through lens distortions. Any sensing must be calibrated so as to, first, accurately determine parameters such as camera points and orientations, and second, to model and allow for as accurately as possible systematic sources of error.

- Accuracy: Optical scanners accuracies typically depend largely on the resolution of the video system used. Distance from the measured surface and accuracy of the moving parts of the scanning system all contribute to the overall measurement error.

- Accessibility: Accessibility is the issue of scanning data that is not easily acquired due to the configuration or topology of the part. This usually requires multiple scans but can also make some data impossible to acquire with certain methods.

- Occlusion and Fixturing: Occlusion is the blocking of the scanning medium due to shadowing or obstruction. This is primarily a problem with optical scanners. However, acoustic and magnetic scanners may also have this problem. Multiple scanning devices are one approach to obviate this problem. The geometry of the fixture is used for scanning the object. Occlusion may also arise due to fixtures-typically parts must be clamped before scanning and sometimes central gravity of the part makes most surfaces of the object difficult to scanning. Multiple views introduce errors in acquired data because of registration problems. 
Kumar, A.; Jain, P. K. \& Pathak, P. M.: Industrial Application of Point Cloud / ST...

- Noise and incomplete data: Noise elimination in data samples is a difficult issue. Noise can be introduced in a multitude of ways, from extraneous vibrations, specular reflections, etc. There are many different filtering approaches and tools that can be used for eliminate the scanned noise. An important issue is whether to eliminate the noise before, after, or during the model building stage.

- Statistical distributions of parts: A similar problem is restoration of missing data. It is partly necessary due to the above-mentioned inaccessibility and occlusion problems. Moreover, the nature of optical and even tactile scanning, the data close to sharp edges is also fairly unreliable. So, there are situations where only parts of a certain surface can be measured. There are missing parts or parts obscured by other elements, but to reconstruct the whole surface from just the visible parts.

- Statistical distributions of parts: Statistical distribution of parts deals with the fact that any given part, which is scanned, only represents one sample in a distributed population. When reverse engineering methods attempt to reproduce a given shape, the tolerance distribution of the scanned part must be considered.

- Surface finish: The final issue is surface finish of the part being measured. Smoothness and material coatings can dramatically affect the data acquisition process. Tactile or optical methods will produce more noise with a rough surface than a smooth one. Reflective coatings also can affect optical methods.

In summary, while systems exist it can perform the simple operation of 3D copying. The goal of extracting higher level information is to be edited and analyzed the data of copying object. Key research areas which still need further work before generalpurpose reverse engineering becomes widely available include: improving data capture and calibration, coping with noise, reliable segmentation, fair surface fitting, recognizing natural structure of the geometry of the object, and finally in this chapter all above steps are discussing with a appropriate problem.

\section{The Present Work-Reverse Engineering for Wear Estimation of Complex Geometry Part}

The present work purpose a technique to identify the wear out portion on spur gear teeth by reverse engineering approach. Spur gear is selected for the study as it is an imperative element in mechanical industries to transmit motion and power between two parallel shafts. During motion and power transfer it worn out with time due to rubbing of meshed gears. Wear may also be caused by foreign element like dust particle, metal debris, etc. Over the span of time, meshed gears geometry of teeth varies continuously and hence, an actual CAD spur gear model is different from its corresponding tattered one. As a result, it can be used to determine whether the service life of gear was over or not. In this study, the spur gear has been scanned before and after the wear using a PICZA 3D laser scanner (Roland LPX60). The scanned data obtained in the form of point cloud or STL data has been used to create 
the surface geometry of spur gear teeth before and after the wear to determine total area of the wear out portion.

\subsection{Data Acquisition}

A data acquisition and processing system includes both hardware and software components. A hardware system acquires point clouds or volumetric data by using available experimental setup. The point cloud data is acquired by scanning of spur gear, after and befor wear shape in form of $\mathrm{x}, \mathrm{y}$ and $\mathrm{z}$ co-ordinates of the multiple point. The scan data is collected with the help of scanning software (Roland LPXEZ studio) and data is saved as a .GSF file format.

\subsection{Generation of Point Cloud Data}

Scanned data of spur gear at every fixed height are required for part modeling or recreation of spur gear teeth profile regardless of density of the scanned data. This cross-sectional data results in a series of stair-steps when a curved feature along the $\mathrm{z}$-axis of a part is built. The data generated during 3D scanning of the spur gear, is in the digital form. It has been saved as Point cloud or STL data and used as output of scanned data. The point cloud data for the scanned gear is shown in Figure 6.

\subsection{Preprocessing}

A modern 3D digitization system such as 3D laser scanner generated the large amount of point cloud as shown in Figure 6. Therefore, before the surface reconstruction process it is necessary to prepare the noise free point cloud data. This preparation is frequently described as preprocessing. In this work, the $3 \mathrm{D}$ editor (version 2.0) tool of Dr. PICZA3 has been used for editing the data and for removing the noise in surface models and noise free data can be saved as point cloud and STL data. The preprocessed image of spur gear is shown in Figure 7.

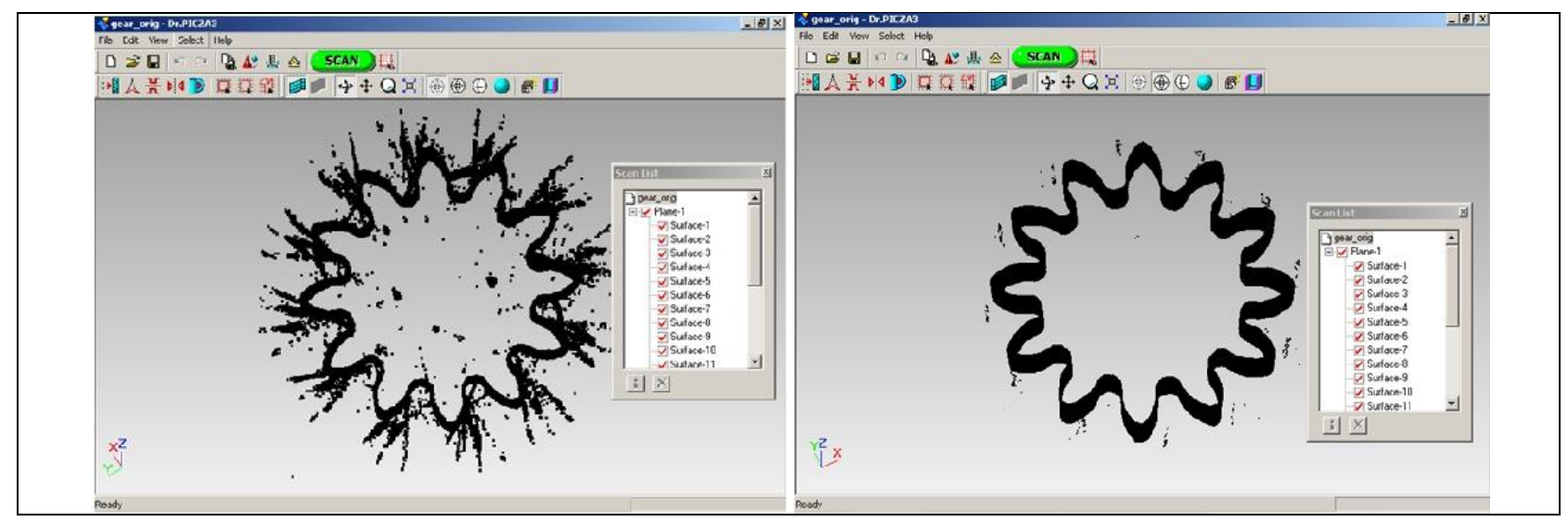

Fig.6. Point cloud data of spur gear

Fig.7. Gear profile after preprocessing

\subsection{Recreation of Spur Gear}

Recreation of model using scanned data can be completed in two ways;

I. Using point cloud data: For recreate, the scanned model Microcal (TM) Origion working model (version 6.0) software has been used. The recreated model before wear is shown in Figure 8 (a), Figure 8 (b) shows the model 
Kumar, A.; Jain, P. K. \& Pathak, P. M.: Industrial Application of Point Cloud / ST...

after wear in three teeth of the gear marked as A, B and C. By comparing Figure 8 (a) and (b) one can estimate the amount of wear at the various gear teeth. For mathematical computation, the gear tooth profiles of tooth B both before and after wear are presented in figure 9 (a).

II. Using STL data: The surface model of the spur gear before and after wear has been created on the Pro/E modeling software. The collected data is converted into surface model. The generated teeth profile has been made such that it best fits with the original teeth profile as shown on figure 8 (d). The generated STL file is imported in Pro/E modeling software by using Insert / shared data / from file / STL. In this way the surface profile of the scanned gear is recreated shown in figure 8 (c). The generated surface model is used for downstream operation on any modeling software such as inspection, compression, analysis, mass properties etc.

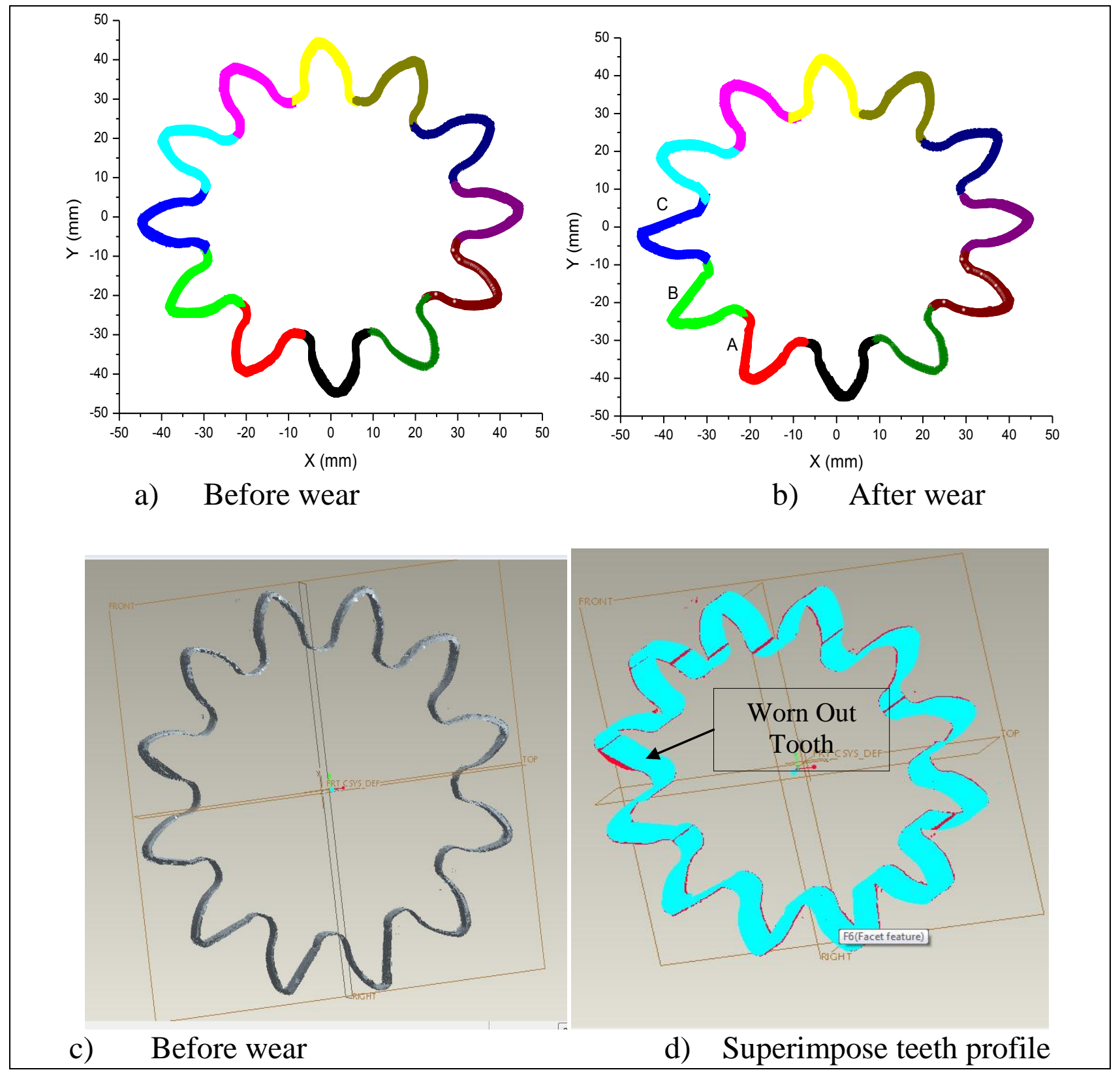

Fig. 8. Recreation of the scanned gear tooth profile 


\subsection{Tooth Profile Comparison}

The tooth profile accuracy also depends on the placement of the gear on the table before and after wear. Hence data is analyzed to extract the point cloud data for different values of $\mathrm{z}(\mathrm{z}=0,1,2 \ldots 14)$. Some minor error may occur during the profile comparison because of scanning process variables like change in voltage, misplacement of gear and variation in rpm while table rotation. The wear out area has been indicated for point cloud data in figure 9 (a) and STL data in figure 9 (b).

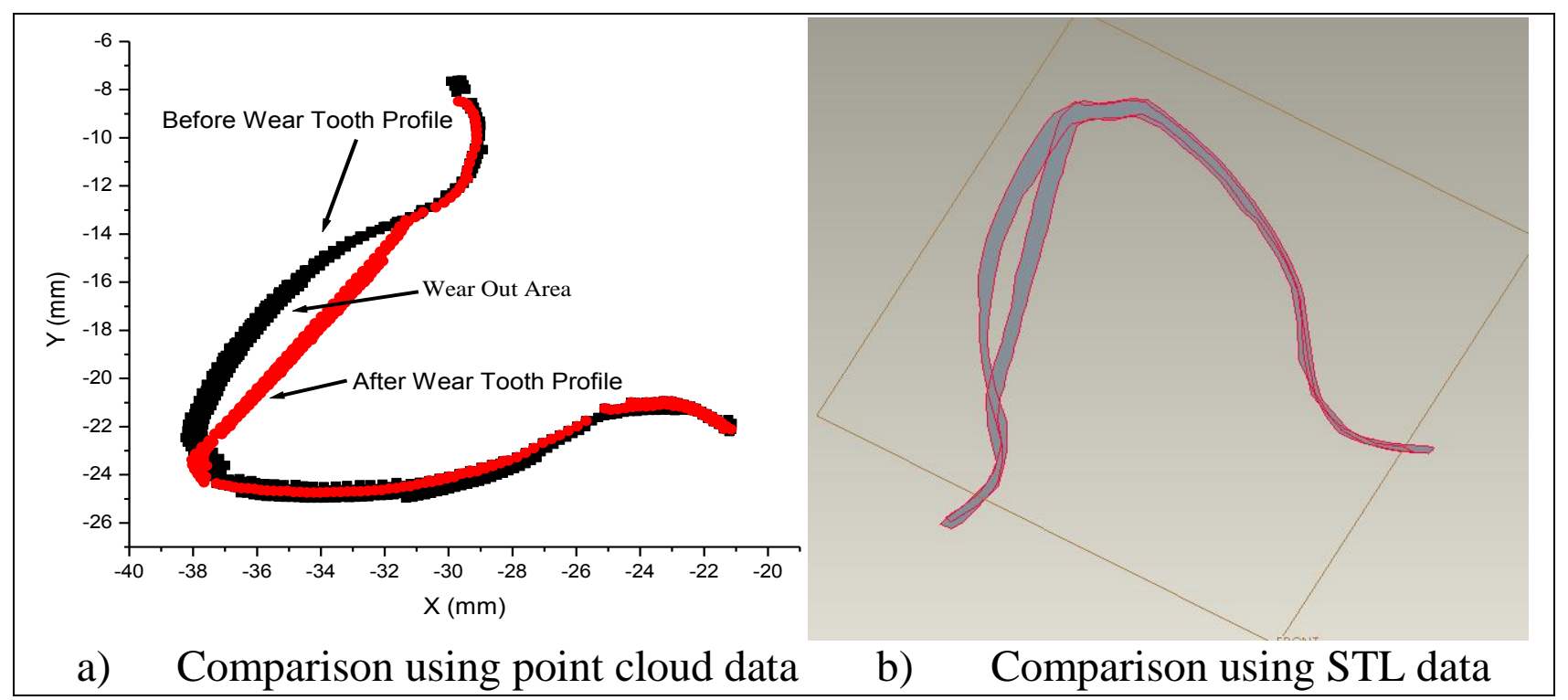

Fig. 9. Wear out area between original and wear out tooth profile

\subsection{Wear out Area on a Tooth}

The wear out area can be measured for any specific value of $\mathrm{z}$ by comparing the tooth profiles both before and after wear. Although the real wear, measurement must take in to account with calculate the area for all values of z (0 to 14). In this work, wear out area has been evaluated using two methods namely; grid technique and pro/E advance tool. Based on grid, area is calculated up to $14 \mathrm{~mm}$. The area has been evaluated, after compared with the original gear geometry using equation 1 . The evaluated area is $283.17 \times 10^{-2}\left(\mathrm{~mm}^{2}\right)$ and that is indicate in table 1 .

$$
\text { Total area }(A)=n i A i+n j A j
$$

Where,

$\mathrm{ni}=$ Number of full square grid

$\mathrm{Ai}=$ Area of full square grid

$\mathrm{nj}=$ Number of half square grid

$\mathrm{Aj}=$ Area of half square grid

The surface CAD model is created using Pro/E modeling software after the processing. The surface fitting operation is carried out for original and worn out gear tooth profile. The wear out area can be measured for all specific value of $\mathrm{z}$ ( 0 to $14 \mathrm{~mm}$ ) by comparing the both tooth profiles before and after wear using Pro/E modeling software. The wear out area of tooth has been evaluated at $\mathrm{z}=0.0 \mathrm{~mm}$ to 
Kumar, A.; Jain, P. K. \& Pathak, P. M.: Industrial Application of Point Cloud / ST...

$14.0 \mathrm{~mm}$ and calculated value is $286.944 \times 10^{-2}\left(\mathrm{~mm}^{2}\right)$ as shown in table 1 . The area is calculated with the help of 14 number of datum planes for individual values of $\mathrm{z}$ using.

\begin{tabular}{|c|c|c|}
\hline \multirow{2}{*}{ Value of $Z(\mathrm{~mm})$} & \multicolumn{2}{|c|}{$\begin{array}{c}\text { Wear Out Area } \\
10^{-2} \times\left(\mathrm{mm}^{2}\right)\end{array}$} \\
\cline { 2 - 3 } & Pro/E Based & Grid Based \\
\hline 0 & 23.319 & 19.12 \\
\hline 1 & 23.040 & 21.56 \\
\hline 2 & 22.199 & 20.43 \\
\hline 3 & 21.778 & 22.02 \\
\hline 4 & 13.093 & 15.12 \\
\hline 5 & 21.764 & 19.85 \\
\hline 6 & 21.548 & 20.02 \\
\hline 7 & 22.071 & 23.45 \\
\hline 8 & 21.232 & 22.68 \\
\hline 9 & 21.232 & 21.68 \\
\hline 10 & 19.529 & 19.20 \\
\hline 11 & 18.713 & 19.76 \\
\hline 12 & 18.713 & 19.76 \\
\hline 13 & 18.713 & 18.52 \\
\hline & Wear out area & Wear out raea \\
\hline & $=286.944 \times 10^{-2}\left(\mathrm{~mm}^{2}\right)$ & $=283.17 \times 10^{-2}\left(\mathrm{~mm}^{2}\right)$ \\
\hline
\end{tabular}

Tab.1. Shows the wear out area at different values of $\mathrm{z}$

\section{Conclusion}

Reverse engineering has changed from a manual procedure to a sophisticated engineering process utilizing modern digitizing technique and advanced CAD systems. In order to obtain adequate CAD model, since surface reconstruction on the basis 3D digitization data often results are in unsatisfactory CAD model, because of this pre-processing of data points before surface reconstruction is needed. The entire study involves object selection, dizidigation techniques, processing, pre-processing, scanned model recreation and CAD model. As a result, the applications of reverse engineering have been greatly extended from the original concept of making an exact copy of a part.

In present work several steps such as, data acquisition, data pre-processing, teeth profile generation and comparison of teeth profile after and before wear has been performed. The wear out portion is calculated in terms of area using two diffrent techniques, for all particular tooth fixed value of $\mathrm{z}$ (i.e. $\mathrm{z}=0,1,2 \ldots \ldots .14$ ). The further research will be carried at to find the total wear on tooth as well as all number of gear teeth in terms of volume reduction after the wear and dizitiged gear suracfe geomatry is to be utilised in CAD/CAM/CAE techiques. In industry, to short the lead time 
demand satisfaction the staics and daynamic study of worn out object can be carried out using $\mathrm{CAD} / \mathrm{CAE}$ techniques.

\section{References}

Bagci, E. (2009). Reverse Engineering Applications for Recovery of Broken or Worn Parts and Re-Manufacturing: Three Case Studies, Journal of Advances in Engineering Software, Vol. 40, (2009), pp 407-418

Bardell, R.; Balendran, V. \& Sivayoganathan, K. (2003). Accuracy Analysis of 3D Data Collection and Free-Form Modeling Methods, Journal of Materials Processing Technology, Vol. 133, (2003), pp. 26-33

Bi, Z M, \& Wang, Lihui (2010). Advances in 3D data acquisition and processing for industrial applications, Robotics and Computer-Integrated Manufacturing, Vol. 26, (2010), pp 403-413

Bopaya, B. I. \& Hosni Y. A.,(1994) Reverse Engineering and Its Relevance to Industrial Engineering: A Critical Review, International journal of Computers and Engineering, Vol. 26, No 2, (1994), pp 343-348

Butler, C. (1991). Investigation Into the Performance of Probes on Coordinate Measuring Machines, Industrial Metrology, Vol. 2, No. 1, (1991), pp 59-70,

Chang, Minho \& Park Sang C., (2008). Reverse engineering of a symmetric object, Computers \& Industrial Engineering, Vol. 55,No. 2, (2008), pp 311-320

Clark, J. (2000). Implementing Non-Contact Digitization Techniques within the Mechanical Design Process, Sensor review, Vol. 20, No. 3, (2000), pp. 195201

Durupt, A.; Remy, S. \& Ducellier, G. (2010). A Knowledge Based Reverse Engineering for Mechanical Components, International journal of Computer Aided Design \& Applications, Vol. 7, No. 2, (2010), pp 279-289

Gadelmawla, E.S. (2011). Computer Vision Algorithms For Measurement and Inspection of Spur Gears, International Journal of Measurement, Vol. 44, (2011), pp 1669-1678

Gao, J. ; Chen X., Zheng, D., Yilmaz ,O. \& Gindy, N. (2006). Adaptive Restoration of Complex Geometry Parts Through Reverse Engineering Application. international Journal of Advances in Engineering Software, Vol. 37, (2006), pp 592-600

Hur, S. M.; Kim, H. C. and Lee, S. H. (2002). STL File Generation with Data Reduction by the Delaunay Triangulation Method in Reverse Engineering, International Journal of Advance Manufacturing Technology, Vol. 19, (2002), pp 669-678

Hussain, M. C.,; Rao, H. S. \& Prasad, K. E. (2008). Reverse Engineering: Point Cloud Generation with CMM for Part Modeling and Error Analysis, ARPN Journal of Engineering and Applied Sciences, Vol. 3, (2008), pp 36-40

Jarvis, R. A. (1983). A Laser Time-of-Flight Range Scanner for Robotic Vision, IEEE PAMI, Vol. 5, No. 5, (1983), pp 505-512 
Kumar, A.; Jain, P. K. \& Pathak, P. M.: Industrial Application of Point Cloud / ST...

Lee, K. H.; Woo, H. \& Suk, T. (2001). Data Reduction Methods for Reverse Engineering, The International Journal of Advanced Manufacturing Technology, Vol. 17, No. 10, (2001), pp735-743

Liang, Chia, Chen, \& Grier, Lin, C.I. (2000). Reverse engineering in the design of turbine blades a case study - a case study in appliying the MAMDP, Robotics and Coputer Integated Manufacturing, Vol. 16, (2000), pp 161-167

Modjarred, A. (1988) Non-Contact Measurement Using a Laser Scanning Probe, Proceedings of the SPIE, pp 229-239, In-Process Optical Measurements, Vol. 1012, (1988)

Moss, J. P.; Linney A. D., Grindrod, S. R., \& Mosse, C. A. (1989). A Laser Scanning System for the Measurement of Facial Surface Morphology, Journal of Optics and Lasers in Engineering, Vol. 10, (1989), pp 179-190

Raja, V. \& Fernandes, K. J. (2008). Reverse Engineering an Industrial Perspective, Springer Series in Advanced Manufacturing, ISBN 978-1-84628-856-2

Sahoo, K. C. \& Menq C. H. (1991). Localization of 3-D Objects Having Complex Sculptured Surfaces Using Tactile Sensing and Surface Description, Journal of Engineering for Industry, Vol. 113, (1991), pp 85-92

Sokovic, M. \& Kopac, J. (2006). RE (reverse engineering) as necessary phase by rapid product development, Journal of Materials Processing Technology, Vol. 175, (2006), pp 398-403

Tai, C. C. and Huang, M. C.(2000). The Processing of Data Points Basing on Design Intent In Reverse Engineering, International Journal of Machine Tools and Manufacture, Vol. 40, (2000), pp 1913-1927

Tsakatikas \& Kaisarlis G. (2007). Reverse Engineering In Industrial Maintenance Component Criticality Analysis, 4th International Conference on NDT, (2007), Chania, Crete-Greece

Tut, V.; Tulcan, A., Cosma, C. \& Serban, I. (2010). Application of CAD/CAM/FEA, Reverse Engineering and Rapid Prototyping in Manufacturing Industry, International Journal of Mechanics, Vol. 4, No. 4, (2010), pp 79-86

Varady; Tamas, Martin, Ralph R. \& Coxt, Jordan (1997). Reverse engineering of geometric models-an introduction, International Journal of Computer-Aided Design. Vol. 29, No 4, (1997), pp 255-268

Woo, H.; Kang E., Wang, S. \& Lee, K. H., (2002). A New Segmentation Method For Point Cloud Data. International Journal of Machine Tools and Manufacture, Vol. 42, (2002), pp 167-178

Xiong, Y. L. (1990). Computer Aided Measurement of Profile Error of Complex Surfaces and Curves: Theory and Algorithm, International Journal of Machine Tools and Manufacturing, Vol. 30, No. 3, (1990), pp 339-357

Yang, W. B.; Chen, M. B. and Yen, Y. N. (2011). An Application of Digital Point Cloud to Historic Architecture in Digital Archives, International Journal of Advances in Engineering Software, Vol. 42, (2011), pp 690-699

Zhou1, J.; Zhou, Q., Yang, L. \& Wu, G.(2006). Acquisition and rendering of characteristic images of the wear surfaces of running parts within machinery, International journal of Advances in Engineering Software, Vol. 37, (2006), pp 592-600 